\title{
NUEVAS PERSPECTIVAS EN CUIDADOS PALIATIVOS
}

\author{
Leo Pessini* y Luciana Bertachini**
}

Resumen: Este artículo examina los cuidados paliativos bajo diversos aspectos. Tras una revisión histórica y conceptual, nos preguntamos cuáles deben ser los cuidados de la salud en los contextos geriátrico y gerontológico, así como su contribución en el mantenimiento y rescate de las capacidades funcionales. Reafirmamos la importancia de la reflexión ética, científica, humana, espiritual y sociocultural sobre los programas dirigidos al cuidado de los ancianos en su multidimensionalidad. Al final, señalamos algunos desafíos referentes a rescatar la sabiduría de vivir y la dignidad en el adiós a la vida.

Palabras clave: cuidados paliativos, ética, medicina paliativa, geriatría, gerontología

\section{NEW PERSPECTIVES IN PALLIATIVE CARE}

\begin{abstract}
This paper examines palliative care under diverse aspects. After a historical and conceptual review, the question of which health care interventions must be done in the geriatric and gerontology context is examined, as well as their contribution to the maintenance and rescue of functional capacities. We reaffirm the importance of ethical, scientific, humane, spiritual and socio cultural reflection about programs addressed to the health care of elderly in their multidimensional coverage. Finally, we point out some challenges with respect to rescuing the wisdom of living and the dignity of dying.
\end{abstract}

Key words: palliative care, ethics, palliative medicine, geriatrics, gerontology

\section{NOVAS PERSPECTIVAS EM CUIDADOS PALIATIVOS}

Resumo: Este artigo apresenta a filosofia dos cuidados paliativos sob diversos aspectos. Inicia com uma revisão histórico conceitual. A seguir nos perguntamos como deveria ser os cuidados palaitivos num contextos geriátrico e gerontológico, bem como sua contribuição na manutenção e resgate das capacidades funcionais. Reafirmamos a importancia da refexão ética, científica, humana, espiritual e socio-cultural nos programas direcionados ao cuidado dos anciãos em sua mutidmensionalidade. Finalmente, apontamos alguns desafios, relacionados com o resgate da sabedoria de viver e a dignidade no adeus à vida.

Palavras chave: cuidados paliativos, ética, medicina paliativa, geriatria, gerontologia

* Profesor Doctor en Teología Moral/Bioética. Vicerrector del Centro Universitario São Camilo, São Paulo. Brasil

** Fonoaudióloga del Ambulatorio de Neuropsiquiatría e Gerontología del Instituto de Geriatría y Gerontología de la Universidad Federal de São Paulo. Brasil

Correspondencia: pessini@scamilo.edu.br 


\section{Introducción}

Datos de la Organización Mundial de la Salud (OMS) estiman que más de 50 millones de personas mueren por año, víctimas de enfermedades fatales; en 2004, 4,9 millones habían sido infectadas con el virus del SIDA; 3,1 millones habían muerto por esa causa y 39,4 millones son portadoras del virus. Cada año fallecen 6 millones de personas a causa del cáncer y hay más de 10 millones de casos nuevos. Se estima que hasta 2020 tendremos 15 millones de nuevos casos al año. Frente a esta realidad, los cuidados paliativos y los hospicios se presentan como una forma innovadora de cuidado en el área de la salud.

La OMS también alienta el aumento de la esperanza de vida en las diversas regiones del mundo como una de las conquistas de la humanidad. Reconoce que el mundo está soportando una transformación demográfica sin precedentes, que el 2050 la población de más de 60 años pasará de 600 millones a 2 mil millones y predice un aumento de $10 \%$ para $21 \%$ del total de la población. El aumento será más grande y más rápido en los países en vías de desarrollo, donde se espera que cuadruplique la población envejecida en los próximos 50 años. En la Declaración Política de la Segunda Asamblea Mundial sobre el Envejecimiento de la ONU (Madrid, 2002) los principios y recomendaciones del Plan de Acción Internacional sobre el Envejecimiento 2002, señalados por la Asamblea General de la ONU 1982, han sido reiterados. Lo mismo aconteció con los principios de la ONU en favor de la gente envejecida, adoptados por la Asamblea General de 1991, que habían definido líneas de acción en las áreas de la independencia, participación, cuidado, dignidad y autorrealización.

Este texto revisa aspectos históricos relacionados con la emergencia de los cuidados paliativos y de los hospicios (I); presenta enseguida la definición de la OMS acerca de estos cuidados (II) y comenta algunos principios de base de esta filosofía (III); introduce luego ciertas cuestiones éticas (IV), aspectos relacionados con la geriatría (V) y, al final, desafíos en términos de la sabiduría del vivir y del morir ${ }^{1}$. Algunos marcos histórico-conceptuales

Desde los tiempos más remotos las sociedades ofrecen ayuda y confortan a sus miembros enfermos y a los que están muriendo. Normalmente, cierta reverencia y una mística profunda se mezclan en estos acontecimientos. El período que sucede a la muerte de seres queridos es marcado generalmente por la ejecución de rituales religiosos y culturales bien definidos, cuyas manifestaciones varían de una cultura a otra.

En la búsqueda de las raíces históricas del movimiento actual de hospicios, descubrimos que en el medioevo el término fue utilizado para describir el lugar que recibía a peregrinos y viajeros: una posada. Más recientemente, desde principios del siglo XIX, ha habido progresos en el cuidado de la gente enferma en fase final. Entre quienes merecen la mención se encuentran: Jeanne Garnier, en Francia; Maria Aikenhead, en Irlanda, y Rose Hawthorne, en EE.UU.

En la mitad del siglo XX, cambios importantes han ocurrido en la medicina occidental y en el ámbito de la salud, como el crecimiento de especializaciones, la creación de nuevos tratamientos y un énfasis creciente en la curación y la rehabilitación. La escena del adiós a la vida, que antes ocurría predominantemente en el ambiente familiar, se transfiere y comienza a suceder en el hospital. Los pacientes en fase final o los casos sin esperanza eran considerados con frecuencia como imperfecciones de la prác-

1 Estas reflexiones incorporan sugerencias críticas de los lectores de Pessini L, Bertachini L. Humanização e cuidados paliativos. São Paulo: Loyola/Centro Universitário São Camilo; 2004. 
tica médica. El interés creciente por la eutanasia en el Reino Unido y Estados Unidos se debe, en gran parte, a la carencia de conocimiento profundo sobre las cuestiones relacionadas con los cuidados al final de la vida.

Al principio de los años 50, en el Reino Unido, la atención se vuelve a la gente en la fase final de la vida, mientras que en Estados Unidos la reacción contra la medicalización de la muerte comenzó a ganar fuerza(1). Cuatro innovaciones pueden ser destacadas: a) la inserción sistemática de preguntas ligadas a los cuidados paliativos en la literatura profesional; b) una nueva visión de los pacientes en fase terminal, que indujo a más estudios acerca del proceso de morir, incluyendo la discusión sobre hasta qué punto los pacientes deben saber de su condición terminal; c) la adopción de una aproximación activa de los cuidados, en oposición a la actitud pasiva tradicional (emergió la determinación de descubrir nuevas e imaginativas maneras de "cuidado" al final de la vida y, más allá de él, el cuidado de los enlutados), y d) una conciencia creciente de la relación entre los estados físicos y los estados mentales trajo un concepto más comprensivo del sufrimiento que desafió las bases sobre las cuales las prácticas médicas hasta entonces se apoyaban.

Fue el trabajo de Cicely Saunders, desarrollado inicialmente en el St. Joseph's Hospice, en Hackney, Londres, el que introdujo una nueva filosofía frente a los cuidados en la fase final de la vida. Con la atención sistemática a las narrativas de los pacientes, la atención cuidadosa a relatos sobre la enfermedad y el sufrimiento se desarrolló el concepto de "dolor total”. Esta visión fue más allá de la dimensión física, englobando las dimensiones social, emocional y espiritual del sufrimiento. Cuando Cicely Saunders estableció el St. Christopher's Hospice, en Londres, en 1967, éste se convirtió inmediatamente en una fuente de inspiración para otras iniciativas de esta naturaleza y, al combinar tres principios clave -cuidados clínicos de calidad, educación e investigación-, pasó a ser una referencia mundial en términos de los cuidados, investigación sobre el dolor y educación para los profesionales de la salud de todo el mundo.

En muchas instituciones que cuidaban pacientes con enfermedades avanzadas e incurables, los esfuerzos para controlar el dolor y otros síntomas no habían avanzado debido a la carencia de comprensión de su naturaleza y a la ineficacia de la medicación. En torno a los años 50 apareció un conjunto importante de drogas, incluyendo psicotrópicos, fenoliasinas, antidepresivos, anti inflamatorios, entre otros. Desde entonces, se tuvo una comprensión más profunda de la naturaleza del dolor y del papel de los opioides en su control.

\section{EI concepto de cuidados paliativos}

El término "paliativo" deriva de pallium, palabra latina que significa "capa", capote. Etimológicamente, significa proporcionar una capa para calentar a "los que pasan frío", toda vez que no pueden más ser ayudados por la medicina curativa. Respecto de la esencia de su concepto, se destaca el alivio de los síntomas, del dolor y del sufrimiento en los pacientes que sufren de enfermedades crónico-degenerativas o están en la fase final, y se trata al paciente en su globalidad de ser y buscando mejorar su calidad de vida.

Estos cuidados no dicen primordialmente relación con los cuidados institucionales, pero consisten básicamente en una filosofía que se puede utilizar en diversos contextos e instituciones, es decir, en el domicilio de la persona, en la institución de salud, en el hospicio o en una unidad específica destinada exclusivamente para este propósito dentro de la institución de salud. 
Según Cicely Saunders, los “cuidados paliativos se han iniciado desde el supuesto que cada paciente tiene su propia historia, relaciones y cultura y de que merece respeto, como un ser único y original. Esto incluye proporcionar el mejor cuidado médico posible y poner a su disposición las conquistas de las últimas décadas, de forma que todos tengan la mejor posibilidad de vivir bien su tiempo"(2). Los cuidados paliativos fueron definidos, entonces, teniendo en cuenta no un órgano, edad, tipo de enfermedad o de patología, sino, ante todo, la evaluación de una diagnosis probable y de posibles necesidades especiales de la persona enferma y de su familia.

Por tradición, fueron considerados como aplicables exclusivamente en momentos de muerte inminente; sin embargo, estos cuidados se ofrecen hoy desde el período inicial del curso de una determinada enfermedad progresiva, avanzada e incurable(3).

En 1987, la medicina paliativa fue reconocida como una especialidad médica, siendo definida como "el estudio y gestión de los pacientes con enfermedad activa, gradual y ultra-avanzada, para la cual es limitado el pronóstico y la aproximación del cuidado es la calidad de vida"(4). Inicialmente, este concepto apareció incorporado a las prácticas médicas. Sin embargo, cuando otros profesionales - como enfermeras, terapeutas ocupacionales, fisioterapeutas, fonoaudiólogos o capellanes- están implicados, se refiere más a cuidados paliativos que a medicina paliativa, porque tal cuidado es casi siempre multiprofesional o interdisciplinario. El Manual de Medicina Paliativa de Oxford, en su tercera edición (2005), aunque hace esta distinción conceptual, utiliza los dos conceptos como sinónimos.

El hospicio, antes de un lugar físico, es un concepto; es una filosofía y un modelo del cuidado, así como una forma organizada de proporcionar atenciones de salud. La filosofía del hospicio amplió su alcance en el transcurso de un tiempo, al incluir a personas que están muriendo no solamente de cáncer, sino de muchas otras enfermedades con pronósticos de menos de seis meses de vida, más allá de quienes pasan por el proceso largo, crónico y imprevisible.

En 1990 la OMS definió cuidados paliativos como "el cuidado activo total de los pacientes cuya enfermedad no responde ya al tratamiento. Tiene prioridad el control del dolor y de otros síntomas y problemas de orden psicológico, social y espiritual. El objetivo de los cuidados paliativos es proporcionar la mejor calidad de la vida para los pacientes y sus familiares"(5).

Esta definición es digna de elogio porque se centra en el paciente, destaca la naturaleza múltiple de la condición humana e identifica la calidad de vida como su objetivo último. El uso del término "curativo" no se justifica, toda vez que muchas condiciones crónicas no pueden ser curadas, pero pueden ser compatibles con una esperanza de vida por algunas décadas.

La OMS definió además, en 1998, los cuidados paliativos para los niños y sus familias -cuyos principios se aplican también a otros trastornos pediátricos crónicos(6). Esta misma organización internacional redefinió en 2002 el concepto acentuando la prevención del sufrimiento(7).

Es importante observar que no tienen que ser considerados esencialmente diferentes de otras formas o áreas de cuidados de la salud. La distinción tornaría difícil y hasta imposible su integración en el curso regular de la atención. Muchos aspectos cruciales de estos cuidados se aplican perfectamente a la medicina curativa, así como, por otra parte, su desarrollo puede influir positivamente sobre otras formas de cuidado de la salud, al valorar aspectos tra- 
dicionalmente subordinados por la medicina tecnocientífica, por ejemplo, la dimensión ético-espiritual de las personas.

\section{Principios de base}

Los cuidados paliativos:

- Valorizan alcanzar y mantener un nivel óptimo de control del dolor y la administración de los síntomas. Esto exige una evaluación cuidadosa de cada persona enferma, considerando su historia detallada, su examen físico y otras indagaciones. La gente enferma debe tener acceso inmediato a toda la medicación necesaria, incluyendo una gama de opioides y de fórmulas farmacéuticas.

- Afirman la vida y entienden el morir como proceso normal. Lo que los seres humanos tenemos en común es la realidad inexorable de la muerte. Los pacientes que solicitan cuidados paliativos no tienen que ser vistos como el resultado de imperfecciones médicas. Estos cuidados tienen como objetivo asegurar a las personas enfermas condiciones que las capaciten y las animen para vivir su vida de una forma útil, productiva y plena hasta el momento de su muerte. La importancia de la rehabilitación, en términos del bienestar físico, psíquico y espiritual, no puede ser descuidada.

- No apresuran ni posponen la muerte; no deben acortar la vida "prematuramente", al igual que las tecnologías de la moderna práctica médica no se aplican para prolongar la vida de forma no natural. No obligan los doctores a emplear indefinidamente tratamientos considerados fútiles o excesivamente onerosos para los pacientes; así también los pacientes pueden rechazar los tratamientos médicos. En cuidados paliativos el objetivo es asegurar la mejor calidad de vida posible y, de ese modo, el proceso de la enfermedad conduce la vida a un extremo natural. Específicamente, la eutanasia y el suicidio asistido no se incluyen en ninguna definición de estos cuidados.

- Integran aspectos psicológicos y espirituales en los cuidados al paciente. Un alto nivel de cuidado físico es, ciertamente, de importancia vital, pero no suficiente en sí mismo. La persona humana no tiene que ser reducida a una simple entidad biológica.

- Ofrecen un sistema de apoyo para ayudar a los pacientes a vivir tan activamente como sea posible hasta el momento de su muerte. El paciente establece los objetivos y las prioridades, y el profesional de la salud debe capacitarlo y atenderlo para alcanzar su objetivo identificado. Las prioridades de un paciente pueden cambiar dramáticamente con el tiempo, pero el profesional debe ser consciente de estos cambios y atender a ellos.

- Ayudan a la familia a ocuparse de la enfermedad del paciente y del duelo. La familia es una unidad de cuidados $\mathrm{y}$, por eso, las dudas y dificultades de sus miembros deben ser identificadas y atendidas. El duelo se inicia antes del momento de la muerte de la persona enferma.

- Exigen trabajo en equipo. Ninguna especialidad por sí misma prepara adecuadamente al profesional para ocuparse de la complejidad de las dudas pertinentes a este período. Aunque el equipo central consiste en un médico, una enfermera y un asistente social, es importante contar con un equipo más grande de profesionales del área médica: fisioterapeutas, terapeutas ocupacionales, entre otros. Para que este grupo trabaje de forma integrada, es crucial establecer metas y objetivos comunes, así como usar medios rápidos y eficaces de comunicación. 
- Buscan mejorar la calidad de vida, concepto que sólo puede ser definido por la persona enferma y que se puede modificar perceptiblemente en el curso del tiempo.

- Son aplicables en el período inicial de la enfermedad y concomitantes con sus modificaciones y con las terapias que prolongan la vida. Se exige entonces que estén íntimamente integrados a los otros servicios de salud, en el hospital o en instituciones comunitarias.

Desde los principios antes descritos se evidencia que los cuidados paliativos se definen no sólo a partir de un tipo determinado de enfermedad, sino que, potencialmente, se aplican a pacientes de todas las edades, basados en una evaluación específica de su diagnóstico y necesidades probables. Además, tienen como foco central alcanzar la mejor calidad de vida posible para cada paciente y su familia, lo que implica la atención específica en lo referente al control de los síntomas y la adopción de un acercamiento holístico que tome en cuenta las experiencias de la vida de la persona y su situación actual. Abarcan la persona que está muriendo y sus prójimos -familiares y amigos-, exigiendo una atención especial en la práctica de una comunicación abierta y sensible con los pacientes, familiares y cuidadores.

\section{Cuestiones éticas}

Las cuestiones éticas implicadas en cuidados paliativos se basan en el reconocimiento de que el paciente incurable - $\mathrm{o}$ en fase terminal- no es un residuo biológico sobre el cual ya no se puede hacer más, y cuya vida no debe ser prolongada innecesariamente. Estamos siempre delante de una persona y, como tal, capaz de relación hasta el momento final y de hacer de la vida una experiencia de plenitud y crecimiento.
Los profesionales deben reconocer los límites de la medicina y prevenir el exceso de tratamiento -la llamada "distanasia"- o el tratamiento fútil. Es importante desafiar la ilusión de que existe solamente una forma de ocuparse del dolor y del sufrimiento: la eliminación de los enfermos. Es necesario acentuar que el llamado "dolor total" -concepto acuñado por Cicely Saunders- no se puede tratar solamente a través de instrumentos técnico-científicos. En el caso del "dolor total", la eficacia de analgésicos se relaciona con la posibilidad de incluir el tratamiento médico en el contexto de relaciones humanas significativas, afectivas.

Los médicos y otros profesionales del cuidado deben respetar la autonomía del paciente, aceptando sus prioridades y sus objetivos, no ocultando la información solicitada por el paciente y respetando sus deseos de no ser tratado cuando esta intervención es sólo una prolongación del proceso de morir. Deben medir cuidadosamente las ventajas del tratamiento (beneficencia) y evaluar los riesgos y beneficios de cada decisión clínica (no-maleficencia) para prevenir el tratamiento fútil(9), que no se condice con los objetivos de la prevención: cura, cuidado, rehabilitación y superación del dolor.

Esta perspectiva de la bioética principialista norteamericana puede ser insuficiente en esta área de los cuidados de la salud. Las éticas del cuidado y de las virtudes se presentan como apropiadas y necesarias en cuidados paliativos. Las primeras enfatizan esencialmente la naturaleza vulnerable y dependiente de los seres humanos, por lo tanto, destacan no sólo el proceso de decidir, sino también la calidad de las relaciones: por ejemplo, continuidad, apertura y confianza. Las éticas de las virtudes critican el acercamiento ético basado en las decisiones desde el carácter, acentuando la importancia de acciones virtuosas $(10)$. 
Los pacientes con enfermedades avanzadas o en estado terminal tienen los mismos derechos básicos que los otros pacientes, por ejemplo, el derecho de recibir los cuidados médicos apropiados, de ayuda personal y de ser informados. Pero, también, les asiste el derecho de rechazar los procedimientos de diagnóstico y/o tratamientos cuando éstos nada agregan frente a la muerte prevista; sin embargo, la denegación del tratamiento no tiene que influir en la calidad de los cuidados paliativos. Más aún, tienen el derecho al grado máximo de respeto por su dignidad y a la mejor analgesia disponible para el dolor y el alivio del sufrimiento.

Aunque la realidad de cada país siempre tiene sus particularidades socio-político-culturales es siempre enriquecedor considerar como fuente de inspiración lo que se hace en otros países. Por ejemplo, lo que la Asociación Húngara de Hospicios y Cuidados Paliativos propone en términos de principios éticos $(8, p .20)$ :

1. Los miembros del equipo de cuidados deben respetar la autonomía de los pacientes al aceptar sus prioridades y objetivos, así como hablar de las opciones del tratamiento. Formular en común el plan de cuidados, nunca ocultar la información que el paciente desea recibir, proveer sus necesidades de información sobre cualquier tratamiento y, también, respetar la opción de abandonarlo.

2. El equipo de cuidados debe evaluar las ventajas y riesgos del tratamiento (beneficencia), evaluar los riesgos en lo referente a las ventajas de cada decisión clínica (nomaleficencia), entender que el paciente tiene derecho al más alto estándar de cuidado en el contexto de los recursos disponibles y juzgar las decisiones en el contexto de la asignación y el uso de recursos (justicia).

3. Los derechos fundamentales de los pacientes que están en el final de la vida son: recibir la asistencia médica necesaria, ser respetado en su dignidad y ser apoyado y cuidado en sus necesidades. Además, tienen derecho al alivio del dolor y del sufrimiento, a ser informados, a la autodeterminación y a la suspensión de tratamientos.

4. El paciente tiene el derecho de recibir información detallada respecto de su estado de salud, sobre cualquiera evaluación médica, examen y oferta de intervenciones en lo que respecta considerar las ventajas, los riesgos y las operaciones potenciales, así como la decisión sobre tales exámenes e intervenciones. Por otra parte, el paciente tiene el derecho de recibir información respecto de cualquier procedimiento o método alternativo, así como del proceso del tratamiento y los resultados esperados.

5. Los pacientes tienen el derecho de participar en las decisiones relacionadas con sus cuidados de salud, es decir, exámenes y tratamiento considerados. El consentimiento informado del paciente es un requisito anterior a cualquier intervención médica.

6. En el caso de que el paciente sufra de una enfermedad considerada incurable y terminal, que -de acuerdo con el conocimiento médico actual- conducirá probablemente a la muerte en un período de tiempo corto, se pueden rechazar éticamente intervenciones de mantenimiento o de rescate de la vida, $y$ dejar que la enfermedad siga su curso natural. En caso de incapacidad, el paciente puede designar a otra persona para el ejercicio de ese derecho. Esta declaración se puede anular en cualquier momento si el paciente así lo desea.

7. De cada acto y decisión se debe dejar constancia en forma escrita. 


\section{Investigación y cuidados paliativos}

Es necesario un pronunciamiento especial respecto de la investigación en cuidados paliativos, toda vez que es parte integrante del desarrollo de estos cuidados. Gracias a la investigación tenemos progresos importantes en el campo del dolor y la administración de los síntomas. Muchas prácticas paliativas, sin embargo, se basan en evidencias históricas y, en muchos casos, sin la necesaria fundamentación con métodos científicos.

El reclutamiento de pacientes para un determinado estudio es difícil y exige mucho tiempo debido a la propia naturaleza de la atención. Por otra parte, existen características clínicas definitivas que complican la investigación en este campo:

- Los pacientes son, con frecuencia, personas viejas que sufren de una cierta condición que afecta muchos sistemas del cuerpo y no solamente un órgano. Esa condición es comúnmente severa y presenta muchos síntomas simultáneos.

- La enfermedad es gradual y sus síntomas cambian muy rápidamente, sobre todo, en los períodos finales.

- El tiempo de supervivencia es limitado y el uso de variada medicación es frecuente.

La investigación en pacientes en fase terminal ha sido éticamente cuestionada, en especial debido a la vulnerabilidad de este grupo de pacientes y de su inhabilidad para participar en el proceso de decisión. Existe alta incidencia de problemas cognitivos y dificultades para lograr su asentimiento libre e informado, toda vez que se produce fácilmente dependencia de la institución en donde son cuidados, sensaciones de gratitud, entre otros elementos.

Tales desafíos no son específicos de esta área, sino también de otras, como la geriatría y la medicina intensiva, pero eso no justifica insertar los cuidados paliativos en una categoría especial. La investigación en esta área debe respetar los principios éticos consagrados internacionalmente, que gobiernan cualquiera investigación clínica. Sin embargo, debe ponerse atención al proceso de evaluación de riesgos y ventajas de un proyecto de investigación determinado, cuya interpretación puede variar mucho según el período de progresión de la enfermedad. Los objetivos del cuidado cambian con frecuencia en los períodos finales $\mathrm{y}$, en éstos, la calidad de vida es la prioridad $\operatorname{mayor}(8, p .30)$.

\section{Cuidados paliativos en geriatría/ gerontología}

El informe europeo de la Organización Mundial de Salud sobre cuidados paliativos afirma enfáticamente que "existen evidencias considerables de que la gente envejecida sufre innecesariamente debido a una carencia de evaluación y de tratamiento generalizados de sus problemas y a la falta de acceso a los programas de cuidados paliativos"(11).

La medicina geriátrica emergió del aumento extraordinario de la esperanza de vida de la población y continúa creciendo en la época actual. Este aumento significativo del tiempo de vida es una gran conquista, atribuida en parte a los avances de la civilización, estándares de vida, salud pública y la creciente intervención de cuidados médicos. La medicina geriátrica reconoció los procesos fisiológicos básicos del envejecimiento, sin embargo, los especialistas médicos en la compañía y cuidado de la gente de avanzada edad necesitaron ir más allá del sistema de la enfermedad y del órgano único - o sea, sobrepasar la óptica de una única especialidad- para considerar la intervención de enfermedades crónicas y medicaciones múltiples, más allá de un estado fisiológico altamente 
cambiante. Hace un cuarto de siglo los geriatras recusaban con frecuencia a los especialistas, alegando que las tecnologías invasivas no beneficiarían a sus pacientes y que éstos y sus familiares preferían un acercamiento más conformista de aceptación de la muerte.

Esta escena se alteró mucho en la actualidad, de tal forma que el papel más frecuente de los geriatras ha sido hablar con los especialistas renuentes o intervencionistas acerca de si, a los 95 años, una mujer tiene una buena posibilidad de beneficiarse de un procedimiento quirúrgico o de una intervención de rehabilitación. La geriatría trabaja más en el proceso de convencer a los médicos que no sean fatalistas cuando están delante de pacientes envejecidos, sino que busquen, de forma asertiva, los problemas tratables, la mejora de la calidad de vida, enfatizando que la base de todo es el paciente en su humanidad y dignidad.

Los cuidados paliativos también son, irónicamente, una "nueva especialidad", que surge del reconocimiento de las conquistas de la tecnología médica moderna. Ésta, aunque ha salvado muchas vidas, no puede irrespetar la dimensión de la mortalidad y de la finitud humanas. Los pioneros en cuidados paliativos reconocen la importancia de las raíces históricas de la medicina y de la enfermería en el cuidado de los moribundos, aliviando el dolor y el sufrimiento, buscando contestar las preguntas e incluyendo a la familia como unidad de cuidados junto con los pacientes en el final de la vida. Este objetivo es hoy tan importante como fue a principios del siglo pasado.

En sus orígenes, estos cuidados se enfocaron principalmente en mejorar la calidad de vida de los pacientes, en los pocos días o semanas restantes de vida, y en respetar la dignidad y la humanidad individuales. En la década pasada ha sido sobrepasada esta perspectiva, pues se considera moralmente relevante sólo cuando "ya nada más se puede hacer para prolongar la vida".

La filosofía de los cuidados paliativos reconoce hoy una transición gradual y la necesidad de equilibrio entre las tentativas legítimas de prolongar la vida -cuando hay posibilidades verdaderas de recuperación- y la atención paliativa de los síntomas y la aceptación de un proceso cuando no hay más cura. Eso es verdad en el cuidado de pacientes en estadios avanzados de enfermedades como Alzheimer, Parkinson, etc.

Actualmente, más del ochenta por ciento de los norteamericanos muere con más de 65 años, aumenta el número de personas con más de 80 años e, incluso, un número expresivo celebra su centenario de vida. La mayoría de esas personas no muere de una forma rápida, característica de un cáncer metastático, sino como consecuencia de la acumulación de enfermedades degenerativas múltiples, más allá de la creciente fragilidad inevitable del proceso biológico humano. Aunque esta trayectoria es menos previsible, sigue muy claramente el pronóstico último.

En el centro de toda la cuestión del aumento de la "esperanza de vida" están el paciente, el médico y el imperativo de establecer una comunicación que permita una relación significativa en uno de los momentos más profundos de la vida. Es grande la responsabilidad del médico para crear una relación con los pacientes y familiares que ayude en la confrontación de esta escena compleja. El cuidador se convierte en un factor clave para ayudar a la gente a encontrar una dirección en el final de la vida.

En una perspectiva integradora, los cuidados paliativos y los valores básicos de la geriatría coinciden: el paciente está en el centro de los cuidados y la perspectiva es interdisciplinaria, holística y comprensiva, más allá del paciente 
y de la familia, vistos como una sola unidad de cuidados. Una prioridad es garantizar, en la medida de lo posible, la independencia funcional y calidad de vida, mediante una evaluación regular y formal que asegure la identificación y el tratamiento de los eventos en el momento adecuado. Ambas especialidades proporcionan cuidados donde el paciente esté, ya sea en su casa o en el hospital, y en cualquier estadio de la enfermedad y cualquiera sea la diagnosis: "El objetivo es dar el cuidado preciso para el paciente preciso y en el momento preciso".

\section{Conclusión}

Los cuidados paliativos constituyen hoy una cuestión importante de la salud pública. Trabajan con el sufrimiento, la dignidad de la persona, el cuidado de las necesidades humanas y la calidad de vida de la gente afectada por una enfermedad crónico-degenerativa o que está en la fase final de la vida. También se preocupan de la ayuda a familias y amigos como unidad de cuidados, frente al sufrimiento por la pérdida, potencial o inminente, de seres queridos.

Nuestro sistema de salud es negligente en lo que respecta a esas necesidades humanas. La próxima etapa será su introducción en el currículo principal de los cursos de medicina y en programas de educación en el área de la salud, sea para los profesionales o para el público en general.

Cicely Saunders afirma que eso requiere "cambio de actitudes y educación de todos los profesionales implicados con los pacientes que tienen una enfermedad crónico-degenerativa. Esto exige un compromiso humano, más allá de medicaciones e intervenciones costosas, y debe ser una preocupación de todos los gobiernos"(2, p.7).

Los cuidados paliativos tienen como objetivo especial aliviar, en los pacientes con enfermedades crónico-degenerativas o en fase terminal, una gama extensa de síntomas de sufrimientos, de orden físico, psíquico, mental y espiritual. Por lo tanto, exigen un equipo multi e interdisciplinario de especialistas, con capacidades específicas y sintonizados entre sí(12-14).

En la medida en que los cuidados paliativos se desarrollan, crece el convencimiento de que con las drogas y las técnicas en la actualidad disponibles es relativamente fácil proporcionar comodidad física al paciente. Sin embargo, aún con eso, la persona puede sentir miedo, soledad o poca autoestima. Los que ahora trabajan en este campo perciben que, además de la atención y control de los síntomas físicos, la filosofía de los cuidados paliativos se preocupa básicamente de la calidad, valor y sentido de la vida.

La investigación confirma que los pacientes que no son informados sienten un mayor sufrimiento físico y psicosocial, y hablan de un nivel inferior de calidad de vida que los que son informados según su voluntad.

En relación con el valor de la vida, a medida que se aproxima su final, las personas se preguntan si su vida ha tenido algún valor para los otros, para la comunidad, y si todavía tienen algún valor como personas, principalmente cuando están incapacitadas por una determinada enfermedad fatal, dependientes de los otros y "siendo" un gasto en términos del cuidado. Investigaciones en EE.UU. han demostrado que la pérdida de independencia y el miedo de convertirse en un peso para los otros, antes que el dolor físico, son, con frecuencia, las primeras motivaciones que llevan la gente a pedir el suicidio asistido o eutanasia. Respetando la evaluación del propio paciente acerca del valor de su vida, mientras aún es consciente del efecto de la depresión o del aislamiento social, estamos frente a uno de los más profundos desafíos clínicos y éticos de los cuidados paliativos. 
Otra preocupación es el sentido de la vida. Únicamente cuando su sufrimiento físico es aliviado y su familia cuida de ella, la persona en fase terminal comienza a hacer preguntas existenciales. Aunque ha disminuido en el mundo occidental secularizado el número de personas que tienen una profunda fe religiosa que confiere sentido a su vida, el 75\% de los pacientes en la fase final expresa el deseo de hablar del sentido de la vida, del sufrimiento y de la muerte, y pueden decepcionarse si nadie se interesa en ayudarlas. Un servicio de pastoral atento a estas necesidades será de gran ayuda en el rescate del sentido de la vida. No es casualidad que el cuidado de las necesidades espirituales y psicosociales esté en el centro de la filosofía de los cuidados paliativos.

El desafío ético, en el contexto de países en vías de desarrollo, debe considerar la cuestión de la dignidad en el adiós a la vida, trascendiendo la dimensión física y biológica y la perspectiva médica del hospital, ampliando el horizonte para integrar el espacio socio-relacional. Hay que hacer entender a la sociedad que morir con dignidad es el resultado de una vida digna, más allá de la mera supervivencia sufri- da. ¿Si no existen condiciones de vida digna, cómo garantizaríamos una muerte digna? Antes de existir un derecho a la muerte humana, hay que destacar el derecho de tener condiciones de conservar y preservar la vida existente y de hacerla desplegar plenamente. Llamaríamos a esto el "derecho a la salud". Es chocante y aun irónico percibir que la misma sociedad que le negó al ser humano el pan para vivir le ofrezca la más avanzada tecnología para que "muera bien".

No podemos aceptar pasivamente la muerte como consecuencia de la indiferencia por la vida, causada por la exclusión, la violencia, los accidentes y la pobreza. La sabiduría nace de la reflexión, de la aceptación y del compromiso con el cuidado de la vida humana en el adiós final. Entre dos límites opuestos: de un lado, la convicción profunda de que no se debe acortar la vida, y del otro, la visión de que no se deben prolongar la agonía, el sufrimiento y la muerte. Entre el acortar la vida y el no prolongarla inútilmente se sitúa el amarás... Así como nos ayudaron a nacer, también necesitamos ser ayudados en el momento del adiós a la vida.

\section{Referencias}

1. Metzger J, Kaplan KO. Transforming Death in America. A State of the Nation Report. Washington, DC: Last Acts; 2001.

2. Saunders C. Preface. In: Davies E, Higginson IJ, (ed.) The solid facts: palliative care. Copenhagen: WHO Regional Office for Europe; 2004.

3. Sepúlveda C. Los cuidados paliativos: perspectiva de la Organización Mundial de la Salud. Dolentium Hominum 2005; 58(1): 16-19.

4. Dolyle D, Hanks G, Cherny NI, Calman K, (ed.) Oxford Textbook of Palliative Medicine. New York: Oxford University Press; 2005: 1.

5. World Health Organization. Cancer Pain Relief and Palliative: a report of a OMS expert committee. Geneva: OMS; 1990.

6. World Health Organization. Cancer pain relief and palliative care in children. Geneva: OMS; 1998. [Website] Available at http://www.who.int/cancer/palliative/definition/en/print.html Último acceso en octubre de 2006. 
7. World Health Organization. National cancer control programmes: policies and managerial guidelines. Geneve: OMS; 2002.

8. Council of Europe. Recommendation Rec 24 of the Committee of Ministers to member states on the organization of palliative care. Adopted on 12 nov 2003: 15-17. [Website] Available at http//www.coc. int. Último acceso en octubre de 2006.

9. Pessini L. Distanásia: até quando prolongar a vida? São Paulo: Loyola/Centro Universitário São Camilo; 2001.

10. Pessini L. Envelhecimento e dignidade humana: ame o(a) idoso(a) que você é ou está nascendo em você! In: Pasqualotti A, Portella MR, Tetinelli LA (orgs.) Envelhecimento humano: desafios e perspectivas. Passo Fundo(RS): Universidade de Passo Fundo; 2004: 311-24.

11. Bertachini L. Envelhecer: desafios, valores e dignidade (vídeo). The 18th congress of the International Association of Gerontology. Rio de Janeiro, June 26-30, 2005.

12. Pessini L. O idoso e a dignidade no processo de morrer. In: Carvalho Filho ET, Papaléo Netto M. Geriatria: fundamentos, clínica e terapêutica. São Paulo: Atheneu; 2005: 755-62.

13. Doyle D, Barnard D. Palliative care and hospice. In: Post SG. (editor in chief) Encyclopedia of bioethics, vol. 4. New York: MacMillan/Thomson/Gale; 2003.

14. Actas de la "XIX Conferencia Internacional sobre los Cuidados Paliativos" (11-13 nov., 2004). Dolentium Hominum 2005; 58(1):8. 